\title{
Nueva formulación tópica de liposomas cargados con acetónido de triamcinolona para prevenir el edema macular quístico asociado a la cirugía de catarata asistida con láser de femtosegundo
}

\section{Novel triamcinolone acetonide-loaded liposomes topical formulation to prevent cystoid macular edema after femtosecond laser-assisted cataract surgery}

\author{
Jose Navarro-Partida1,2, Gerardo D. Jauregui-García ${ }^{1}$, Juan C. Altamirano-Vallejo ${ }^{1,2}$, Ricardo Acosta-González², \\ Alejandro González-De la Rosa ${ }^{1,2}$, Juan Armendáriz-Borunda ${ }^{1,3}$ y Arturo Santos ${ }^{1,2 *}$ \\ ${ }^{1}$ Tecnológico de Monterrey, Escuela de Medicina y Ciencias de la Salud, Campus Guadalajara, Zapopan; ${ }^{2}$ Centro de Retina Médica y Quirúrgica, \\ Centro Médico Puerta de Hierro, Zapopan; ${ }^{3}$ Instituto de Biología Molecular y Terapia Génica, Centro Universitario de Ciencias de la Salud, \\ Universidad de Guadalajara, Guadalajara. Jal., México
}

\section{Resumen}

Objetivo: El objetivo de este estudio es informar la tolerabilidad, la seguridad y la eficacia de una nueva formulación de liposomas de uso tópico cargados con acetónido de triamcinolona (FL-AT), útil en la prevención del edema macular clínicamente significativo (EMCS) que ocurre después de la cirugía de catarata asistida con láser de femtosegundo (FLACS). Métodos: Se estudiaron 55 ojos de 32 pacientes a los que se realizó FLACS. Se asignaron 27 ojos al grupo de acetónido de triamcinolona (AT), mientras que 28 ojos fueron asignados al grupo de FL-AT. En el grupo de AT, los ojos se expusieron a una formulación tópica convencional de AT al $0.1 \%$ durante 21 días tras la cirugía, mientras que los pacientes en el grupo FL-AT recibieron una formulación liposomal que contenía $2 \mathrm{mg} / \mathrm{ml}$ de AT (0.2\%). Se realizó un seguimiento clínico que comprendió: examen con lámpara de hendidura, agudeza visual, sensibilidad al contraste, grosor foveal central y volumen macular total. Las visitas del estudio se programaron los días 1 y 6 y al final de la semana 12 del periodo postoperatorio. Resultados: La incidencia de EMCS en el grupo de AT a las 6 semanas fue del 22.2\%, en contraste con el grupo de FL-AT, en el cual fue de 0\%. La formulación de esteroides convencionales (AT al 0.1\%) se asoció con un mayor riesgo de EMCS (OR: 9.44; IC del 95\%: 1.76-50.66; $p=0.027$ ). Finalmente, no se reportaron eventos adversos oculares ni sistémicos en ningún grupo. Conclusión: La FL-AT es eficaz para la prevención del EMCS asociado con FLACS, y parece que su actividad terapéutica es superior a la actividad de la formulación de esteroides tópicos convencionales.

Palabras clave: Liposomas. Acetónido de triamcinolona. Edema macular. Cirugía de catarata asistida con láser de femtosegundo.

Correspondencia:

*Arturo Santos

Ciencias de la Salud, Tecnológico de Monterrey

Campus Guadalajara

Av. General Ramón Corona, 2514

Zapopan, Jalisco, México

Fecha de recepción: 08-05-2019

Fecha de aceptación: 06-05-2020

a-mail: arturo.santos@ DOI: 10.24875/RMO.M20000126
Disponible en internet: 01-09-2020 Rev Mex Oftalmol. 2020;94(5):193-203

www.rmo.com.mx 0187-4519/@ 2020 Sociedad Mexicana de Oftalmología. Publicado por Permanyer. Este es un artículo open access bajo la licencia CC BY-NC-ND (http://creativecommons.org/licenses/by-nc-nd/4.0/). 


\section{Abstract}

Aim: The aim of this study is to report the tolerability, safety and efficacy of a topical triamcinolone acetonide-loaded liposomes formulation (TA-LF) to prevent clinically significant macular edema (CSME) after femtosecond laser-assisted cataract surgery (FLACS). Methods: Fifty-five eyes of 32 patients who underwent FLACS were enrolled. Twenty-seven eyes were assigned to the TA group whereas twenty-eight eyes were assigned to the TA-LF group. In the TA group, eyes were exposed to a conventional topical formulation of triamcinolone acetonide $0.1 \%$ for 21 days postoperatively, whereas patients in the TA-LF group received a liposomal formulation containing $2 \mathrm{mg} / \mathrm{ml}$ of TA $(0.2 \%)$. A follow-up consisting on slit-lamp examination, visual acuity, contrast sensitivity, central foveal thickness (CFT) and total macular volume (TMV) (both measured by retinal optical coherence tomography) was performed. Study visits were scheduled at 1 day, 6 , and 12 weeks after surgery. Results: The incidence of CSME in the TA group at 6 weeks was 22.2\%, in contrast, the incidence of these finding in the TA-LF group was 0\%. A conventional steroid formulation (TA 0.1\%) was associated with an increased risk of CSME (OR, 9.44; 95\% Cl, $1.76-50.66 ; p=0.027)$. Finally, no ocular or systemic adverse events were reported in any group. Conclusion: TA-Ioaded liposomal formulation is effective for the prevention of CSME associated with FLACS, and it seems that its therapeutic activity could be superior to the activity of a conventional topical steroid formulation.

Key words: Liposomes. Triamcinolone acetonide. Macular edema. Femtosecond laser-assisted cataract surgery.

\section{Introducción}

Hoy en día, la catarata sigue siendo la causa número uno de ceguera tratable y discapacidad visual severa en todo el mundo'. La cirugía de catarata mejora la agudeza visual en la mayoría de los pacientes, y se considera una de las intervenciones de atención médica con una gran relación costo-eficacia². La cirugía de catarata asistida con láser de femtosegundo (FLACS) parece mejorar los resultados y la seguridad en comparación con la facoemulsificación convencional, y ha ganado popularidad entre los cirujanos de catarata. En un estudio reciente que compara FLACS y facoemulsificación convencional, ambas técnicas tuvieron resultados refractivos y visuales comparables. Sin embargo, FLACS mostró un menor uso de energía de facoemulsificación, menos inflamación postoperatoria de la cámara anterior y menor pérdida de células endoteliales corneales ${ }^{3}$.

Las técnicas actuales de cirugía de catarata reducen significativamente las complicaciones postoperatorias. Sin embargo, el edema macular cistoide pseudofáquico (EMCP) sigue siendo la causa más común de disminución de la agudeza visual central (AVC) después de una cirugía de catarata exitosa. El EMCP clínico se define por una pérdida visual sintomática, y su incidencia varía de $1.17-4.04 \%$. Sin embargo, la incidencia de EMCP diagnosticado mediante tomografía de coherencia óptica (OCT) puede ser tan alta como $10.9 \%{ }^{5}$. Estudios previos han mostrado menos inflamación de la cámara anterior y un menor grosor macular central con FLACS en comparación con la facoemulsificación convencional ${ }^{6-8}$, sin embargo, se ha reportado EMCP con FLACS. La prevalencia reportada de edema macular cistoide (EMC) postoperatorio asociado con FLACS es de alrededor del $0.8 \%{ }^{9}$, y es comparable con los valores publicados previamente para la cirugía de catarata mediante facoemulsificación convencional $(0.1-2.35 \%)^{10,11}$.

El EMCP clínicamente significativo generalmente comienza de 4 a 12 semanas después de la cirugía, con la incidencia más alta a las 4 a 6 semanas. Los pacientes con EMCP clínicamente significativo comúnmente experimentan una mejora de la agudeza visual postoperatoria inicial, seguida de una reducción de la visión central $^{12}$. Los factores de riesgo conocidos para EMCP incluyen diabetes mellitus ${ }^{4,13}$, capsulotomía YAG, uveítis ${ }^{4,14}$, uso de análogos de prostaglandinas tópicos ${ }^{15,16}$, trauma $^{17}$ y complicaciones transoperatorias ${ }^{18,19}$.

La patogénesis del EMCP es compleja e implica la producción de prostaglandinas (PG), citocinas y otros factores liberados durante el trauma quirúrgico, que alteran la barrera hematorretiniana ${ }^{12}$. Las herramientas terapéuticas disponibles para la profilaxis y el tratamiento del EMCP se basan en la comprensión de estos factores y sus vías. No se ha establecido el mejor régimen profiláctico para el EMCP, pero los corticosteroides y los antiinflamatorios no esteroideos (AINE) tópicos, solos o combinados, han demostrado ser útiles ${ }^{20-23}$, y se utilizan ampliamente como medicamentos de primera línea ${ }^{24}$. Un estudio integral previo que evaluó la prevención del EMCP después de la cirugía de catarata, demostró que la terapia tópica con AINE (bromfenaco al 0.09\%) o esteroides (dexametasona al $0.1 \%$ ) solos, se relacionó con una mayor incidencia de EMCP clínicamente significativo 12 semanas después de la cirugía (incidencia de 3.6 y $5.1 \%$, respectivamente) en comparación con la terapia combinada (incidencia del $1.5 \%)^{25}$. 
La mayor eficacia de la combinación tópica podría estar relacionada con los diferentes mecanismos de acción de los esteroides y los AINE. Los corticosteroides bloquean la síntesis de leucotrienos y PG y reducen la migración de macrófagos y neutrófilos, así como la permeabilidad capilar y la vasodilatación. Por otro lado, los AINE inhiben las enzimas de la ciclooxigenasa, bloqueando la síntesis de PG y tromboxanos, lo cual disminuye la vasodilatación y evita la alteración de la barrera hematoocular ${ }^{24}$. La actividad terapéutica reducida de los corticosteroides tópicos podría explicarse por la concentración limitada del fármaco que llega al segmento posterior del ojo debido a la presencia de las barreras ocular y hematorretiniana. Por otro lado, las inyecciones intravítreas de esteroides logran concentraciones intraoculares adecuadas, ya que evitan las barreras oculares ${ }^{26}$.

El EMCP crónico y refractario se puede manejar con éxito con corticosteroides intravítreos. El acetónido de triamcinolona intravítreo (ATIV) mejora efectivamente la agudeza visual y disminuye el grosor macular ${ }^{27-30}$. Sin embargo, el ATIV se asocia con complicaciones potencialmente graves, como aumento de la presión intraocular ${ }^{27,30}$, endoftalmitis, lesión del cristalino, desprendimiento de retina ${ }^{31-33}$, formación o progresión de cataratas y endoftalmitis no infecciosa ${ }^{34-36}$.

Existe la necesidad de estrategias alternativas de administración de fármacos para el EMCP que minimicen los riesgos de las inyecciones intravítreas pero que sean tan efectivas como el ATIV. Recientemente, informamos sobre una formulación tópica de liposomas cargados con acetónido de triamcinolona (FL-AT) que administra eficazmente triamcinolona en el vítreo y la retina de $\operatorname{conejos}^{37}$, y su actividad biológica y terapéutica también se confirmó en pacientes con EMCP refractario ${ }^{38}$, mostrando que el uso de esta formulación indujo una mejora de la agudeza visual mejor corregida (AVMC) y del grosor foveal central (GFC). Además, recientemente publicamos la comparación de la FL-AT con AINE $^{39}$. En este estudio, demostramos que la FL-AT se correlaciona con mejores resultados de sensibilidad al contraste en comparación con la terapia combinada (prednisolona al $1 \%$ y nepafenaco al $0.1 \%$ ) después de FLACS. Es interesante notar que no se observaron diferencias en la incidencia de edema macular clínicamente significativo (EMCS) entre los grupos $^{39}$. En el estudio actual, decidimos comparar la FL-AT con suspensiones oftálmicas de esteroides convencionales, para informar la evaluación de tolerabilidad, seguridad y eficacia de la FL-AT tópica para la prevención del EMCP después de FLACS.

\section{Pacientes y métodos}

\section{Diseño del estudio}

Este fue un estudio prospectivo, aleatorizado, doble ciego, de dos grupos, en un solo centro (Centro de Retina Medica y Quirúrgica SC, Guadalajara, México). El estudio se adhiere a los principios de la Declaración de Helsinki y las aprobaciones del Comité de Ética del hospital, y los acuerdos regulatorios locales y federales (COFEPRIS 173300410A0035/2017). Todos los pacientes que se sometieron al procedimiento e ingresaron al estudio firmaron un consentimiento informado, y todos los datos se recolectaron prospectivamente en formularios estandarizados de informes de casos.

\section{Pacientes}

Se incluyeron pacientes con edades de 21 años 0 más que requerían FLACS e implante de lentes de cámara posterior en al menos un ojo. Se recopilaron los datos demográficos y de un examen clínico basal que incluyó la medición de GFC mediante OCT, de 1 a 14 días antes de la cirugía. Los criterios de exclusión incluyeron el uso de esteroides tópicos o AINE tópicos 1 mes antes del estudio, la colocación de un implante ocular de esteroides 12 meses antes del reclutamiento en el estudio, el uso de corticosteroides intraoculares o medicamentos antiangiogénicos 3 meses antes del estudio, catarata traumática u opacidad nuclear o color del cristalino mayor a grado 3 (según el sistema de clasificación de opacidades del cristalino III [LOCS III]). También excluimos a los pacientes con enfermedades oculares que impedían un examen adecuado del fondo de ojo, cualquier enfermedad ocular que pudiera disminuir la agudeza visual (retinopatía diabética, oclusión vascular, degeneración macular), hipertensión ocular, glaucoma, enfermedad sistémica inestable, incluida la hipertensión sistémica, diabetes mellitus, enfermedad ocular previa que resultara en un historial médico de EMC. También se excluyeron los sujetos con accidente cerebrovascular previo o infarto de miocardio. Solo se incluyeron pacientes que se sometieron a cirugía de catarata sin complicaciones.

\section{TÉCNICAS QUIRÚRGICAS Y TERAPIA ASIGNADA}

Todos los pacientes recibieron el mismo tratamiento preoperatorio y transoperatorio en ambos ojos. Todas las cirugías se realizaron bajo anestesia tópica utilizando clorhidrato de tetracaína al 5\% (Ponti Ofteno, 
Sophia Labs., Zapopan, Mex.). Para mantener la dilatación de la pupila durante toda la cirugía, se utilizaron tropicamida al $0.8 \%$ y clorhidrato de fenilefrina al $5 \%$ (TP Ofteno, Sophia Labs., Zapopan, Mex.). Los pacientes se sometieron a FLACS, y se aplicó la parte con láser del procedimiento primero. El sistema de láser LenSX (Alcon, Inc., EE.UU.) se utilizó para realizar la capsulotomía anterior, la fragmentación del cristalino y luego las incisiones corneales (incisión temporal principal y dos puertos laterales de paracentesis), en esa secuencia. Primero se aplicó un anillo de succión. Se creó una capsulotomía circular con la energía establecida en $9 \mu \mathrm{J}$. El diámetro de la capsulotomía fue de $5.0 \mathrm{~mm}$. Se utilizó un patrón combinado circular (2 círculos, diámetros de $2.0 \mathrm{~mm}$ y $2.8 \mathrm{~mm}$ ) y 4 cortes en forma de cruz (diámetro de $8.0 \mathrm{~mm}$ ) para la fragmentación del cristalino con una energía de pulso de $10 \mu \mathrm{J}$. El láser de femtosegundo se utilizó para crear dos puertos de paracentesis y una incisión corneal clara. El ancho de la incisión corneal del puerto principal fue de $2.2 \mathrm{~mm}$, y de $1.0 \mathrm{~mm}$ para cada uno de los 2 puertos laterales. El puerto principal se colocó a $210^{\circ}$ en el ojo derecho y a $30^{\circ}$ en el ojo izquierdo. Los puertos laterales estaban a $95^{\circ}$ y $275^{\circ}$ en el ojo derecho, y a $100^{\circ}$ y $280^{\circ}$ en el ojo izquierdo.

Después del tratamiento con láser de femtosegundo, los pacientes fueron transferidos a la sala de operaciones para la facoemulsificación. Después de la sedación, se abrieron las incisiones corneales y la cápsula anterior separada se retiró con fórceps. Todos los demás pasos fueron similares a la facoemulsificación convencional. Todos los pacientes recibieron el lente intraocular corrector de presbicia AcrySof ${ }^{\circledR} \quad I Q$ PanOptix ${ }^{\mathrm{TM}}$ de Alcon.

Después de la cirugía, los pacientes fueron asignados aleatoriamente a 1 de 2 grupos de tratamiento. Los pacientes en el grupo de acetónido de triamcinolona (AT) recibieron gotas de acetónido de triamcinolona al $0.1 \%$ disponibles comercialmente en México (Softram, Laboratorios Grin, SA de CV; 03-04-2008 / 087 M2008 SSA / IPP: DEAR 05330060101527 / R-2008) cuatro veces al día durante 21 días después de la cirugía, mientras que los pacientes en los grupos de FL-AT recibieron una formulación liposomal que contiene 2 $\mathrm{mg} / \mathrm{ml}$ de AT ( $0.2 \%)$ cuatro veces al día durante 21 días después de la cirugía. No se permitieron otros corticosteroides oculares o AINE durante el curso del estudio. Todos los pacientes recibieron gatifloxacino al $0.3 \%$ después de la cirugía, cuatro veces al día durante 14 días (Zymar, Allergan, Irvine, CA, EE.UU.).

\section{Evaluación de eficacia}

Para evaluar la eficacia terapéutica de FL-AT al 0.2\% en comparación con AT al $0.1 \%$, se realizó un seguimiento que incluyó agudeza visual, sensibilidad al contraste, GFC y volumen macular total (VMT). La AVMC se midió utilizando la cartilla del ETDRS (del inglés, Early Treatment Diabetic Retinopathy Study) a 4 m, y la agudeza visual medida se expresó como el logaritmo del ángulo mínimo de resolución (logMar). La sensibilidad al contraste (SC) se evaluó mediante la prueba de sensibilidad al contraste de Pelli-Robson. Los valores logarítmicos de sensibilidad al contraste (1/contraste), así como los valores de agudeza visual, se registraron en cada visita del estudio. El análisis de GFC y VMT se realizó mediante OCT (Cirrus OCT Carl Zeiss, Meditec, Dublin, CA). Las visitas del estudio se programaron el día 1 y las semanas 6 y 12 después de la cirugía. Además, se realizaron tinción de la superficie ocular con fluoresceína, medición de presión intraocular (PIO) y evaluación del segmento anterior y posterior con lámpara de hendidura en cada visita, con el propósito de identificar cualquier evento adverso ocular.

La aparición de EMC y EMCS se exploró en las semanas 6 y 12. El EMC se definió como un aumento del grosor macular medio del subcampo central del $10 \%$ o más sobre el valor basal, con cambios quísticos mediante OCT. Los cambios cistoides y otros trastornos de la retina fueron identificados por dos especialistas en retina independientes y enmascarados. El EMCS se definió como EMC con una mejora de menos de 0.2 logMAR de AVMC en comparación con la basal preoperatoria ${ }^{25}$.

\section{Evaluación de seguridad}

La tolerabilidad se evaluó mediante la recopilación y el Resumen de eventos adversos (EA) oculares y no oculares, EA serios (EAS), evaluaciones oculares y signos vitales, referidos espontáneamente por los pacientes reclutados, descubiertos por el personal del sitio de estudio durante el interrogatorio o por otros medios. Los sujetos eran retirados si mostraban alguna evidencia de mala tolerabilidad o algún evento adverso, como úlceras corneales, opacidades corneales, defectos epiteliales, inflamación de la cámara anterior (células/flare) o inyección conjuntival y/o epiescleral relacionada con el uso de esta formulación tópica. Los EA se codificaron utilizando los términos de códigos estándar para el evento con base en el Diccionario 
médico para la Terminología de Actividades Regulatorias (MedDRA) versión 18.1.

\section{TRATAMIENTO DE RESCATE}

Se consideró el tratamiento de rescate con una combinación tópica de prednisolona al 1\% 4 veces al día y nepafenaco al $0.1 \% 3$ veces al día durante 4 semanas, cuando los pacientes desarrollaban EMCS durante el transcurso del estudio. Si el EMCS se resolvía dentro de estas 4 semanas, se suspendía el nepafenaco y las gotas de prednisolona se disminuían gradualmente 1 gota por día cada semana. Si el EMCS persistía después de 4 semanas de tratamiento tópico, los pacientes recibían una inyección intravítrea de $4 \mathrm{mg}$ de AT sin conservantes. Se consideraba el uso de fármacos reductores de la PIO cuando la $\mathrm{PIO} \geq 22 \mathrm{mmHg}$ o si existía una diferencia $>4 \mathrm{mmHg}$ en comparación con el ojo contralateral.

\section{Preparación de la formulación liposomal}

OPKO Health, Inc. (Guadalajara, Jalisco, México) proporcionó una formulación de liposomas cargados con FL-AT. La preparación de FL-AT se realizó como se describió previamente ${ }^{37}$. Brevemente, se generaron espontáneamente liposomas cargados con AT termodinámicamente estables (QuSomes ${ }^{\circledR}$ ) al agregar polietilenglicol (PEG-12) gliceril dimiristato a una solución acuosa de AT. La composición de la FL-AT se describe en la tabla 1. La concentración final de AT en la dispersión resultante fue de $2 \mathrm{mg} / \mathrm{ml}(0.2 \%)$.

\section{Análisis estadístico}

Los datos se analizaron utilizando el software SPSS 22.0 (IBM SPSS Statistics para Macintosh, Versión 22.0. Armonk, NY, EE.UU.: IBM Corp.). Las variables cuantitativas se describieron utilizando media y desviación estándar. Las variables cualitativas se describieron utilizando frecuencias y porcentajes. Realizamos la prueba de los rangos con signo de Wilcoxon y la prueba $U$ de Mann-Whitney para el análisis de la edad, AVMC, SC, GFC, VMT y PIO en muestras dependientes e independientes, respectivamente. Para el análisis del género y el ojo del estudio, se realizó una prueba exacta de Fisher. La correlación entre las variables se midió mediante la $r$ de Pearson para calcular posteriormente el coeficiente de determinación $\left(r^{2}\right)$. La significancia se definió como un valor de p menor de 0.05 .
Tabla 1. Composición de la formulación de liposomas cargados con acetónido de triamcinolona

\begin{tabular}{|l|c|}
\hline Reactivo & Volumen \\
\hline Acetónido de triamcinolona & $2.0 \mathrm{mg}$ \\
\hline Kolliphor HS 15 & $50 \mathrm{mg}$ \\
\hline PEG-12 gliceril dimiristato & $100 \mathrm{mg}$ \\
\hline Alcohol etílico & $14 \mu \mathrm{L}$ \\
\hline Ácido cítrico anhidro & $0.8 \mathrm{mg}$ \\
\hline Citrato de sodio dihidrato & $4.675 \mathrm{mg}$ \\
\hline Cloruro de benzalconio & $0.1 \mathrm{mg}$ \\
\hline Agua purificada de grado 2 & Afore a $1.0 \mathrm{ml}$ \\
\hline PEG: polietilenglicol & \\
\hline
\end{tabular}

Tabla 2. Características clínicas de los grupos

\begin{tabular}{|c|c|c|c|}
\hline & AT & FL-AT & Valor p \\
\cline { 1 - 3 } & $n=27$ & $n=28$ & \\
\cline { 1 - 2 } Edad & $57.25 \pm 7.0$ & $58.39 \pm 5.7$ & 0.5099 \\
\hline $\begin{array}{c}\text { Género } \\
\text { F }\end{array}$ & $15(0.55)$ & $18(0.64)$ & 0.5088 \\
M & $12(0.45)$ & $10(0.36)$ & \\
\hline Ojo & & & \\
OD & $13(0.48)$ & $13(0.46)$ & 0.8983 \\
OI & $14(0.52)$ & $15(0.54)$ & \\
\hline
\end{tabular}

AT: grupo acetónido de triamcinolona; F: femenino; FL-AT: grupo de formulación liposomal que contiene $2 \mathrm{mg} / \mathrm{ml}$ de AT; OD: ojo derecho; Ol: ojo izquierdo.

\section{Resultados}

Se reclutaron 55 ojos de 32 pacientes. De los 55 ojos del estudio, 29 eran derechos y 26 eran izquierdos. La proporción hombre:mujer de los pacientes reclutados fue de 15:17. Fueron asignados al grupo de AT 27 ojos, mientras que 28 ojos fueron asignados al grupo de FL-AT. Solo 21 ojos del grupo de AT completaron 12 semanas de seguimiento, ya que seis pacientes requirieron tratamiento de rescate en la semana 6 debido a EMCS. Todos los pacientes del grupo de FL-AT finalizaron el seguimiento. La figura 1 muestra un diagrama de flujo con el número de ojos aleatorizados y analizados. Las características clínicas de los pacientes en los grupos de AT y FL-AT se Resumen en la tabla 2.

Con respecto a los resultados de tolerabilidad y seguridad, observamos que la FL-AT fue bien tolerada durante el periodo de estudio. No se informaron EA 


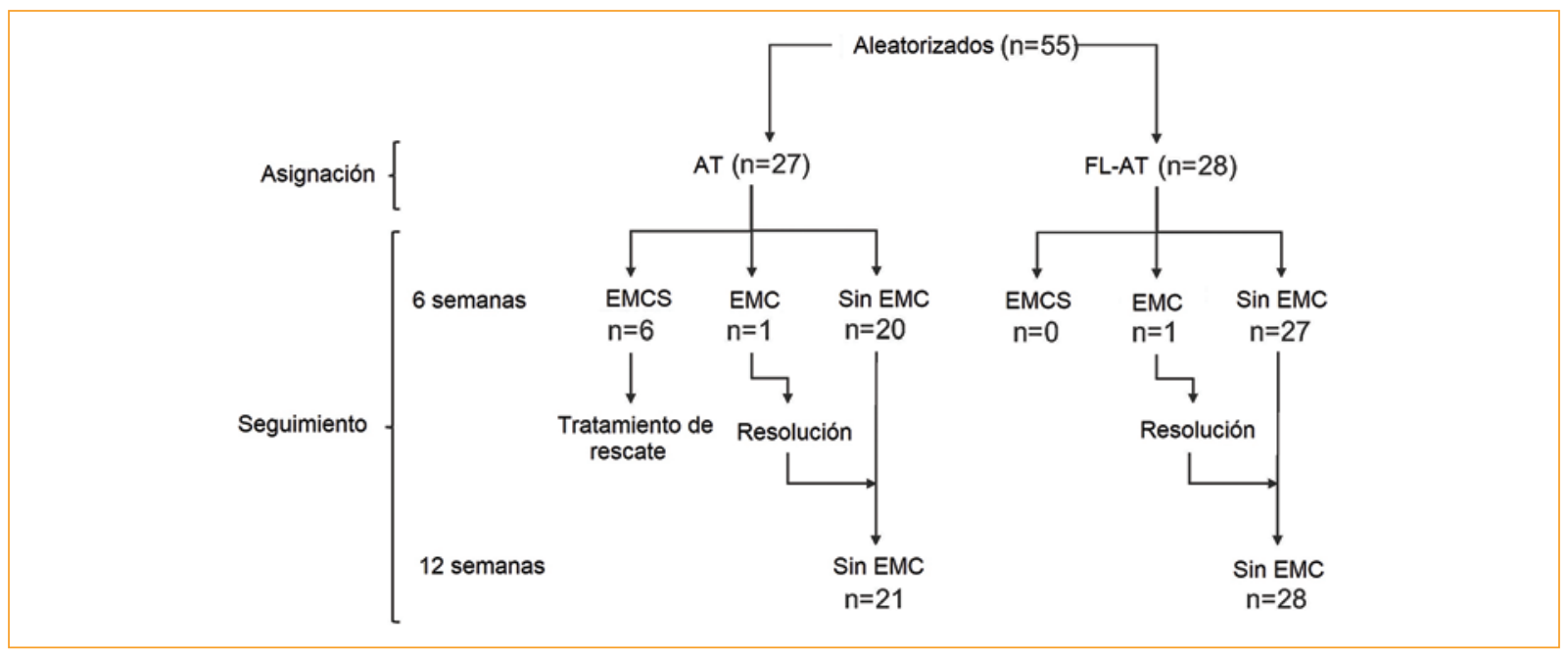

Figura 1. Diagrama de flujo con el número de ojos aleatorizados y analizados.

AT: grupo de acetónido de triamcinolona; EMC: edema macular cistoide; EMCS: edema macular clínicamente significativo; FL-AT; grupo de formulación liposomal que contiene 2 mg/ml de AT.

Tabla 3. Diferencias dentro de los grupos de agudeza visual, sensibilidad al contraste, grosor macular y volumen macular total

\begin{tabular}{|l|l|l|l|l|l|}
\hline & & Basal & $6 \mathrm{~s}$ & $12 \mathrm{~s}$ & Valor p \\
\hline Agudeza visual (logMAR) & AT & $0.054 \pm 0.148$ & $0.072 \pm 0.189$ & $0.077 \pm 0.195$ & 0.9779 \\
\hline & FL-AT & $0.252 \pm 0.248$ & $0.03 \pm 0.142$ & $0.005 \pm 0.136$ & $<0.0001$ \\
\hline Sensibilidad al contraste (1/contraste) & AT & $1.214 \pm 0.219$ & $1.194 \pm 0.173$ & $1.229 \pm 0.178$ & 0.513 \\
& FL-AT & $1.087 \pm 0.339$ & $1.217 \pm 0.191$ & $1.266 \pm 0.147$ & 0.0346 \\
\hline GFC (mm) & AT & $258.33 \pm 32.50$ & $275.37 \pm 43.22$ & $275.38 \pm 47.26$ & 0.0143 \\
\hline VMT (mm) & FL-AT & $256.21 \pm 15.16$ & $266.42 \pm 16.06$ & $265.92 \pm 18.55$ & $<0.0001$ \\
\hline PIO (mmH) & AT & $10.14 \pm 0.70$ & $10.41 \pm 0.58$ & $10.52 \pm 0.69$ & 0.031 \\
\hline & FL-AT & $10.08 \pm 0.71$ & $10.55 \pm 0.52$ & $10.50 \pm 0.52$ & $<0.0001$ \\
\hline & AT & $15.11 \pm 3.25$ & $12.88 \pm 3.02$ & $13.52 \pm 2.50$ & 0.0193 \\
\hline
\end{tabular}

AT: grupo de acetónido de triamcinolona; FL-AT: grupo de la formulación liposomal que contiene $2 \mathrm{mg} / \mathrm{ml}$ de AT; GFC: grosor foveal central; logMAR: logaritmo del ángulo mínimo de resolución; PIO: presión intraocular; s: semana; VMT: volumen macular total.

oculares (aumento de la presión intraocular, anomalías de la superficie ocular) o sistémicos. Ninguno de los pacientes mostró un aumento significativo de la PIO. De hecho, se observó una reducción significativa de la PIO en los grupos de AT y FL-AT. Los niveles de PIO disminuyeron de $15.11 \pm 3.25$ a $12.88 \pm 3.02$ en la semana 6 en el grupo de AT, y de $15.71 \pm 2.20$ a 12.89 \pm 2.69 en la semana 6 en el grupo de FL-AT (Tabla 3). Ninguno de los pacientes requirió fármacos reductores de la PIO. Ninguno de los pacientes mostró signos de irritación o problemas de superficie debido a la formulación del estudio hasta el final del estudio (Fig. 2).

Por otro lado, como se esperaba, hubo un aumento significativo del GFC y del VMT postoperatorios, en comparación con los valores preoperatorios, en ambos grupos; sin embargo, solo el grupo de FL-AT mostró una mejora significativa de la sensibilidad al contraste (valor basal; $1.087 \pm 0.339$ frente a la semana 12; $1.266 \pm$ 0.147 ) y de la agudeza visual en comparación con las mediciones preoperatorias (valor basal; $0.252 \pm 0.248$ 


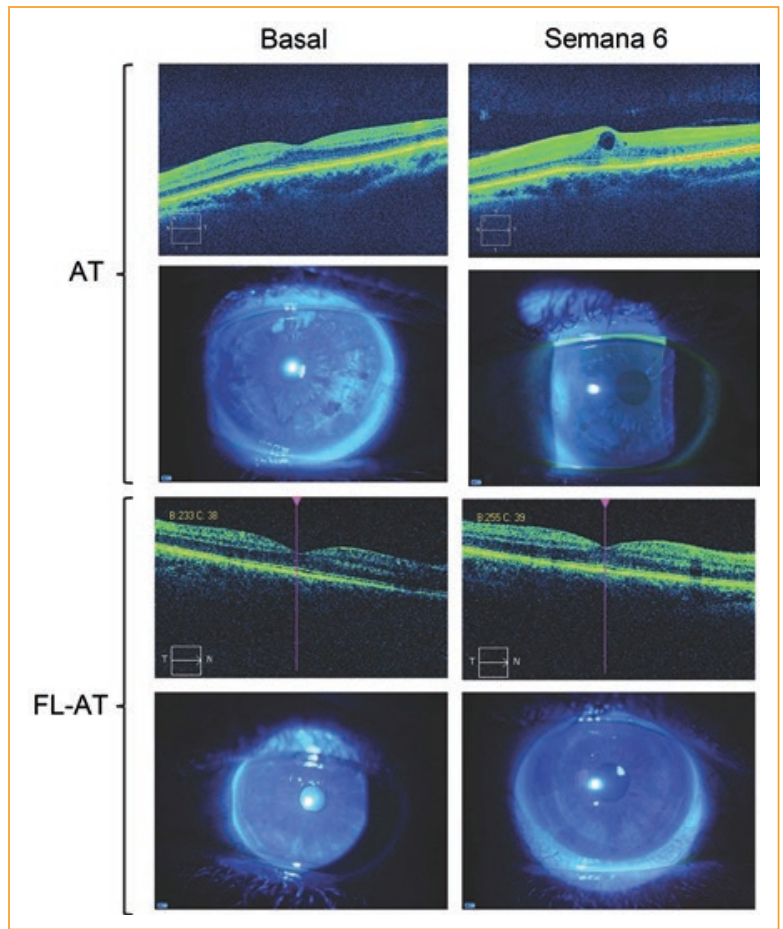

Figura 2. Casos representativos. Imágenes basales y postoperatorias de tinción con fluoresceína e imágenes de OCT en los grupos de AT y FL-AT. Las imágenes tomográficas en el grupo de AT corresponden a uno de los seis casos con EMCS, mientras que las imágenes tomográficas en el grupo de FL-AT corresponden al único caso de EMC. Como mostramos en las fotografías, no se observaron eventos adversos en la superficie ocular mediante tinción con fluoresceína a las 6 semanas de seguimiento en ningún grupo.

AT: grupo de acetónido de triamcinolona; EMC: edema macular cistoide; EMCS: edema macular clínicamente significativo; FL-AT: grupo de formulación liposomal que contiene $2 \mathrm{mg} / \mathrm{ml}$ de AT.

frente a la semana $12 ; 0.005 \pm 0.136$ ). La tabla 3 resume el análisis de variables dentro de los grupos.

El análisis de eficacia entre los grupos mostró que los pacientes tratados con FL-AT tuvieron el grosor macular postoperatorio más bajo y la mejor AVMC y sensibilidad al contraste después de la corrección para las mediciones basales (Tabla 4); sin embargo, estos hallazgos no fueron estadísticamente diferentes en comparación con el grupo de AT. No hubo diferencias estadísticamente significativas del VMT y la PIO entre los grupos de AT y FL-AT en ningún caso. Las diferencias entre los grupos en agudeza visual, SC, grosor macular, VMT y PIO se Resumen en la tabla 4.

Es importante mencionar que el GFC y el VMT se correlacionan significativamente con la sensibilidad al contraste solo en el grupo de FL-AT. El $r^{2}$ para el GFC y la sensibilidad al contraste fue de $0.1675(p=0.0306)$, mientras que el $r^{2}$ para el VMT y la SC fue de 0.1675 $(p=0.0055)$ (Tabla 5).

Finalmente, encontramos que la FL-AT mostró la mejor acción preventiva para el EMCS. Las incidencias de EMC y EMCS en el grupo de AT a las 6 semanas fueron de 3.7 y $22.2 \%$, respectivamente, mientras que la incidencia en el grupo de FL-AT fue de $3.7 \%$ y $0 \%$ (Tabla 6). Las probabilidades de desarrollar EMCS fueron significativamente mayores en el grupo de AT que en el grupo de FL-AT (OR: 9.44; IC 95\%, 1.76 -50.66; $p=0.027$ ). Todos los pacientes con EMCS en el grupo de AT requirieron tratamiento de rescate. La incidencia de EMC se mantuvo sin cambios en los grupos de AT y FL-AT durante el estudio, y no fue estadísticamente significativa en todos los puntos de tiempo (Tabla 6). Las imágenes de OCT de un caso representativo de EMCS en el grupo de AT y las imágenes de OCT del único caso de EMC en el grupo de FL-AT se presentan en la figura 2.

\section{Discusión}

El EMCP continúa siendo la complicación postoperatoria más frecuente que resulta en deterioro visual después de la cirugía de catarata. Aunque es casi siempre autolimitado ${ }^{40}$, los casos persistentes y refractarios representan un desafío terapéutico ${ }^{13}$. Se ha utilizado una amplia gama de agentes farmacológicos para el tratamiento del EMCP, incluidos los esteroi$\operatorname{des}^{29,41,42}$ y AINE ${ }^{43-45}$. Los corticosteroides son potentes moléculas antiangiogénicas y antiinflamatorias que desempeñan un papel importante en el tratamiento de diferentes enfermedades vitreorretinianas debido a su capacidad para regular la expresión de genes clave como el VEGF y la interleucina- $6^{46,47}$. Sin embargo, los corticosteroides tópicos tienen baja penetración al segmento ocular posterior debido a las barreras ocular y hematorretiniana. Por otro lado, las inyecciones intravítreas de esteroides logran concentraciones intraoculares adecuadas ya que evitan las barreras oculares ${ }^{26}$. El AT intravítreo ha demostrado ser adecuado para el tratamiento del EMCP refractario y de otras enfermedades vitreorretinianas ${ }^{27-30,48-50}$. Sin embargo, para disminuir los riesgos oculares relacionados con las inyecciones intravítreas de AT y para preservar los beneficios de su uso en el EMCP refractario, es necesario desarrollar estrategias alternativas para la administración de medicamentos. 
Tabla 4. Diferencias dentro de los grupos en agudeza visual, sensibilidad al contraste, grosor macular y VMT

\begin{tabular}{|c|c|c|c|c|}
\hline & Parámetro & AT & FL-AT & Valor $\mathbf{p}$ \\
\hline \multirow[t]{3}{*}{ Agudeza visual (logMAR) } & Basal & $0.054 \pm 0.148$ & $0.252 \pm 0.248$ & 0.000791 \\
\hline & $6 \mathrm{~s}$ & $0.072 \pm 0.189$ & $0.03 \pm 0.142$ & 0.349688 \\
\hline & $12 \mathrm{~s}$ & $0.077 \pm 0.195$ & $0.005 \pm 0.136$ & 0.135063 \\
\hline \multirow[t]{3}{*}{ Sensibilidad al contraste (1/contraste) } & Basal & $1.214 \pm 0.219$ & $1.087 \pm 0.339$ & 0.105633 \\
\hline & $6 \mathrm{~s}$ & $1.194 \pm 0.173$ & $1.217 \pm 0.191$ & 0.637007 \\
\hline & $12 \mathrm{~s}$ & $1.229 \pm 0.178$ & $1.266 \pm 0.147$ & 0.437292 \\
\hline \multirow[t]{3}{*}{$\mathrm{GFC}(\mu \mathrm{m})$} & Basal & $258.333 \pm 32.504$ & $256.214 \pm 15.164$ & 0.756522 \\
\hline & $6 s$ & $275.37 \pm 43.229$ & $266.428 \pm 16.065$ & 0.310517 \\
\hline & $12 \mathrm{~s}$ & $275.38 \pm 47.267$ & $265.928 \pm 18.553$ & 0.338887 \\
\hline \multirow[t]{3}{*}{ VMT $\left(\mathrm{mm}^{3}\right)$} & Basal & $10.14 \pm 0.707$ & $10.085 \pm 0.711$ & 0.774737 \\
\hline & $6 \mathrm{~s}$ & $10.414 \pm 0.584$ & $10.557 \pm 0.52$ & 0.343936 \\
\hline & $12 \mathrm{~s}$ & $10.52 \pm 0.697$ & $10.503 \pm 0.52$ & 0.886292 \\
\hline \multirow[t]{3}{*}{ PIO (mmHg) } & Basal & $15.11 \pm 3.25$ & $15.71 \pm 2.2$ & 0.424754 \\
\hline & $6 \mathrm{~s}$ & $12.88 \pm 3.02$ & $12.89 \pm 2.69$ & 0.989694 \\
\hline & $12 \mathrm{~s}$ & $13.52 \pm 2.5$ & $13.5 \pm 1.93$ & 0.974905 \\
\hline
\end{tabular}

AT: grupo acetónido de triamcinolona; FL-AT: grupo de formulación liposomal que contiene $2 \mathrm{mg} / \mathrm{ml}$ de AT; GFC: grosor foveal central; logMAR: logaritmo del ángulo mínimo de resolución; PIO: presión intraocular; s: semana; VMT: volumen macular total.

Tabla 5. Correlación entre el GFC y el VMT con la agudeza visual y la sensibilidad al contraste en los grupos de AT y FL-AT

\begin{tabular}{|c|c|c|c|c|c|}
\hline & & \multicolumn{2}{|c|}{$\begin{array}{l}\text { Agudeza visual } \\
\text { (logMAR) }\end{array}$} & \multicolumn{2}{|c|}{$\begin{array}{c}\text { Sensibilidad al } \\
\text { contraste (1/contraste) }\end{array}$} \\
\hline & & $r^{2}$ & Valor de p & $r^{2}$ & Valor de $\mathrm{p}$ \\
\hline \multirow[t]{2}{*}{ AT } & GFC & 0.04249 & 0.3023 & 0.01382 & 0.854 \\
\hline & VMT & 0.00008 & 0.9629 & 0.01236 & 0.5809 \\
\hline \multirow[t]{2}{*}{ FL-AT } & GFC & 0.1036 & 0.0948 & 0.1675 & 0.0306 \\
\hline & VMT & 0.06301 & 0.1976 & 0.2605 & 0.0055 \\
\hline
\end{tabular}

AT: grupo de acetónido de triamcinolona; FL-AT: grupo de formulación liposomal que contiene $2 \mathrm{mg} / \mathrm{ml}$ de AT; GFC: grosor foveal central; logMar: logaritmo del ángulo mínimo de resolución; s: semana. VMT: volumen macular total,

Un estudio farmacocinético reciente de una nueva formulación tópica de FL-AT informó que esta formulación es capaz de liberar AT de manera eficiente en el vítreo y la retina ${ }^{37}$. Además, un estudio clínico reciente en pacientes con EMCP refractario mostró que la FL-AT es eficaz para reducir el GFC y mejorar la AVMC en pacientes con esta afección. Fue bien tolerado con un perfil de seguridad adecuado y sin eventos adversos oculares observados o cambios significativos de la
$\mathrm{PIO}^{38}$. Estos hallazgos sugieren que la FL-AT tópica podría ser efectiva en el tratamiento de pacientes con EMCP refractario, y que tiene el potencial de sustituir a los esteroides intravítreos. Estos antecedentes nos llevaron a evaluar la eficacia de la FL-AT en la prevención del EMCP en este ensayo clínico. Como se muestra, la FL-AT tuvo la mejor acción preventiva para el EMCS después de FLACS cuando esta formulación se compara con una formulación de esteroides tópicos convencionales (AT al 0.1\%). La incidencia de EMCS en el grupo de AT a las 6 semanas fue de $22.2 \%$, en contraste con la incidencia en el grupo de FL-AT de $0 \%$. Como se puede observar, la incidencia de EMCS en el grupo de AT es perceptiblemente mayor en comparación con la incidencia en el grupo de FL-AT. Suponemos que este fenómeno está relacionado con la mayor capacidad de la FL-AT para llegar al vítreo y la retina y liberar AT en dosis terapéuticas. Esta suposición se basa en la equivalencia de los grupos de AT antes y después de FLACS. Además, la incidencia de EMCS después de FLACS (22.2\%) difiere mucho en comparación con la incidencia previa de EMCS $(0.8 \%)$ informada por Ewe, et al. ${ }^{9}$. Es posible que la mayor incidencia observada en nuestra serie se deba a la 
Tabla 6. Incidencia de los valores de EMC y EMCS y relación de probabilidades

\begin{tabular}{|c|c|c|c|c|}
\hline \multirow[t]{2}{*}{ Parámetro } & AT & FL-AT & \multirow[t]{2}{*}{ OR (IC $95 \%$ )* } & \multirow[t]{2}{*}{ Valor de $\mathrm{p}$} \\
\hline & Incidencia $\mathrm{n} / \mathrm{N}(\%)$ & Incidencia n/N (\%) & & \\
\hline EMC dentro de $6 \mathrm{~s}$ & $1 / 27(3.7)$ & $1 / 28(3.5)$ & $1.038(0.0617-17.48)$ & 0.97 \\
\hline EMC dentro de $12 \mathrm{~s}$ & $1 / 21(4.7)$ & $1 / 28(3.5)$ & $1.33(0.078-22.57)$ & 0.84 \\
\hline EMCS dentro de $6 \mathrm{~s}$ & 6/27 (22.2) & $0 / 28(0)$ & $9.44(1.76-50.66)$ & 0.027 \\
\hline EMCS dentro de $12 \mathrm{~s}$ & $0 / 21(0)$ & $0 / 28(0)$ & $1.325(0.025-69.52)$ & 0.88 \\
\hline
\end{tabular}

AT: grupo de acetónido de triamcinolona; Cl: intervalo de confianza; EMC: edema macular cistoide; EMCS: edema macular clínicamente significativo; FL-AT: grupo de formulación liposomal que contiene $2 \mathrm{mg} / \mathrm{ml}$ de AT; OR: relación de probabilidades.

Se presentan las posibilidades de desarrollar EMC y EMCS en el grupo de AT.

definición operativa de EMCS utilizada en nuestro estudio $^{25}$. Ewe, et al., definieron el EMCS como una agudeza visual deficiente inesperada (es decir, falta de mejora postoperatoria) 0 deterioro de la agudeza visual (en comparación con visitas anteriores), ya sea en el examen de 3 a 4 semanas o en cualquier visita posterior, con evidencia de EMC mediante examen de fondo de ojo, OCT de dominio espectral (SD-OCT) o angiografía con fluoresceína ${ }^{9}$. Por otro lado, nosotros utilizamos la definición de EMCS propuesta en el ensayo multicéntrico europeo de la prevención del EMC después de cirugía de catarata en no diabéticos (ESCRS PREMED). Este ensayo es un gran estudio multicéntrico de gran impacto para evaluar la acción preventiva de los AINE y los esteroides como monoterapia o terapia combinada para el EMCP ${ }^{25}$. En este trabajo, la definición de EMCS fue edema macular (aumento del grosor macular medio del subcampo central de $10 \%$ o más en comparación con el valor basal, con cambios quísticos mediante SD-OCT), con una mejora de menos de 0.2 logMAR de AVMC en comparación con el valor basal preoperatorio ${ }^{25}$.

Además, el uso de la formulación convencional de esteroides aumentó el riesgo de EMCS, con un OR de 9.44 (IC de 95\%: 1.76 -50.66; $p=0.027$ ) cuando esta solución se comparó con la nueva FL-AT. Estos resultados sugieren que la FL-AT supera la actividad terapéutica de la formulación convencional de esteroides tópicos. Curiosamente, el aumento de la PIO, un efecto adverso común de los esteroides tópicos, no se observó en ningún grupo tratado con la formulación de esteroides tópicos convencionales o la formulación de esteroides liposomales. Quizás el tiempo de uso del medicamento fue relativamente corto (15 días) para inducir este efecto no deseado. De hecho, la PIO disminuyó significativamente después de la cirugía de catarata. Este fenómeno no es inesperado, ya que es bien sabido que la cirugía de catarata reduce la presión intraocular en alrededor de $1.5 \mathrm{a} 4 \mathrm{mmHg}^{51}$. El mecanismo de este efecto no está claro, pero parece estar relacionado con los cambios anatómicos y fisiológicos inducidos por la cirugía de cristalino ${ }^{52}$.

En comparación con estudios previos que utilizan otros esteroides tópicos, la FL-AT sigue siendo terapéuticamente superior. Por ejemplo, un estudio retrospectivo que comparó los esteroides tópicos para la prevención del EMCP (definido como edema macular anatómico nuevo o que empeora, o engrosamiento demostrado mediante OCT) informó una diferencia no significativa en la tasa de EMC postoperatorio en pacientes que reciben acetato de prednisolona al $1 \%$ y fosfato sódico de dexametasona al $0.1 \%(4.0 \text { vs. } 4.1 \%, p=0.94)^{22}$, mientras que en un gran estudio prospectivo, la incidencia de EMCS fue de $5.1 \%$ para el fosfato sódico de dexametasona al $0.1 \%$. En cualquier caso, la incidencia de EMC es menor que con $\mathrm{FL}^{-A T^{25}}{ }^{2}$, presumiblemente debido al uso de liposomas.

Las gotas oculares basadas en liposomas se han propuesto como un nuevo sistema de administración de medicamentos para el segmento posterior del ojo, con el potencial de administrar medicamentos a concentraciones terapéuticas en la cavidad vítrea y la retina $^{37}$. Los liposomas son partículas compuestas de un núcleo acuoso delimitado por una bicapa lipídica similar a una membrana que actúa como portador de fármacos anfifílicos, solubles en lípidos y solubles en agua ${ }^{53-56}$. Los LP no son tóxicos, son poco antigénicos, se metabolizan fácilmente y son biodegradables $^{57}$, y se han utilizado para mejorar el transporte de fármacos y la biodisponibilidad en los tejidos oculares ${ }^{58,59}$. Los hallazgos de este estudio respaldan el potencial de los liposomas para aumentar la biodisponibilidad (con base en su eficiencia terapéutica superior), así como su seguridad, ya que ninguno de 
los pacientes mostró signos de irritación o problemas en la superficie, ni aumentos significativos de PIO secundarios a la formulación del estudio. Sin embargo, se deben realizar estudios complementarios para confirmar estos hallazgos.

Es interesante notar que los pacientes tratados con FL-AT tuvieron el grosor macular postoperatorio más bajo y la mejor AVMC y sensibilidad al contraste después de la corrección para las mediciones basales (Tabla 3); sin embargo, estos hallazgos no fueron estadísticamente significativos en comparación con el grupo de AT. Es posible que en estudios posteriores con tamaños de muestra más grandes, se puedan lograr diferencias significativas. Esta posibilidad es de gran interés para la cirugía de cristalino moderna, especialmente en la cirugía de cristalino para presbicia, donde el objetivo principal es la calidad visual.

Finalmente, hay varias limitaciones en nuestro estudio. Primero, la falta de un grupo control es una preocupación importante, pero debido a consideraciones éticas, no se consideró un grupo placebo. La segunda limitación importante de nuestro estudio es el pequeño tamaño de la muestra.

En conclusión, la formulación liposómica cargada con AT es efectiva para la prevención del EMCS asociado con FLACS, y parece que su actividad terapéutica podría ser superior a la actividad de las formulaciones de esteroides tópicos convencionales. Sin embargo, se debe considerar realizar más estudios.

\section{Conflicto de intereses}

Los autores declaran no tener ningún conflicto de interés.

\section{Responsabilidades éticas}

Protección de personas y animales. Los autores declaran que los procedimientos seguidos se conformaron a las normas éticas del comité de experimentación humana responsable y de acuerdo con la Asociación Médica Mundial y la Declaración de Helsinki.

Confidencialidad de los datos. Los autores declaran que han seguido los protocolos de su centro de trabajo sobre la publicación de datos de pacientes.

Derecho a la privacidad y consentimiento informado. Los autores han obtenido el consentimiento informado de los pacientes y/o sujetos referidos en el artículo. Este documento obra en poder del autor de correspondencia.

\section{Bibliografía}

1. Bourne RR, Stevens GA, White RA, Smith JL, Flaxman SR, Price $H$. et al. Causes of vision loss worldwide, 1990-2010: a systematic analysis. Lancet Glob Health. 2013;1:e339-49.

2. Sight. WHOVTRt. Global Initiative for the Elimination of Avoidable Blindness; Action Plan 2006-2011. En: WHO (ed.). Geneva, Switzerland: WHO; 2007. Disponible en: http://appswhoint/iris/bitstream/10665/43754/ 1/9789241595889_engpdf.

3. Ang RET, Quinto MMS, Cruz EM, Rivera MCR, Martinez GHA. Comparison of clinical outcomes between femtosecond laser-assisted versus conventional phacoemulsification. Eye Vis (Lond). 2018;5:8.

4. Chu CJ, Johnston RL, Buscombe C, Sallam AB, Mohamed Q, Yang YC. Risk Factors and Incidence of Macular Edema after Cataract Surgery: A Database Study of 81984 Eyes. Ophthalmology. 2016;123:316-23.

5. Perente I, Utine CA, Ozturker C, Cakir M, Kaya V, Eren $\mathrm{H}$, et al. Evaluation of macular changes after uncomplicated phacoemulsification surgery by optical coherence tomography. Curr Eye Res. 2007;32:241-7.

6. Ecsedy M, Mihaltz K, Kovacs I, Takács A, Filkorn T, Nagy ZZ. Effect of femtosecond laser cataract surgery on the macula. J Refract Surg. 2011; 27:717-22

7. Nagy ZZ, Ecsedy M, Kovacs I, Takács Á, Tátrai E, Somfai GM, et al. Macular morphology assessed by optical coherence tomography image segmentation after femtosecond laser-assisted and standard cataract surgery. J Cataract Refract Surg. 2012;38:941-6.

8. Abell RG, Allen PL, Vote BJ. Anterior chamber flare after femtosecond laser-assisted cataract surgery. J Cataract Refract Surg. 2013;39:1321-6.

9. Ewe SY, Oakley CL, Abell RG, Allen PL, Vote BJ. Cystoid macular edema after femtosecond laser-assisted versus phacoemulsification cataract surgery. J Cataract Refract Surg. 2015;41:2373-8.

10. Henderson BA, Kim JY, Ament CS, Ferrufino-Ponce ZK, Grabowska A, Cremers SL. Clinical pseudophakic cystoid macular edema. Risk factors for development and duration after treatment. J Cataract Refract Surg. 2007;33:1550-8

11. Zur D, Loewenstein A. Postsurgical Cystoid Macular Edema. Dev Ophthalmol. 2017:58:178-90.

12. Coscas G, Cunha-Vaz J, Soubrane G. Macular Edema: Definition and Basic Concepts. Dev Ophthalmol. 2017;58:1-10.

13. Schmier JK, Halpern MT, Covert DW, Matthews GP. Evaluation of costs for cystoid macular edema among patients after cataract surgery. Retina. 2007;27:621-8.

14. Belair ML, Kim SJ, Thorne JE, Dunn JP, Kedhar SR, Brown DM, et al. Incidence of cystoid macular edema after cataract surgery in patients with and without uveitis using optical coherence tomography. Am J Ophthalmol. 2009;148:128-135 e122.

15. Yeh PC, Ramanathan S. Latanoprost and clinically significant cystoid macular edema after uneventful phacoemulsification with intraocular lens implantation. J Cataract Refract Surg. 2002;28:1814-8.

16. Panteleontidis V, Detorakis ET, Pallikaris IG, Tsilimbaris MK. Latanoprost-Dependent Cystoid Macular Edema Following Uncomplicated Cataract Surgery in Pseudoexfoliative Eyes. Ophthalmic Surg Lasers Imaging. 2010;1-5.

17. Rossetti L, Autelitano A. Cystoid macular edema following cataract surgery. Curr Opin Ophthalmol. 2000;11:65-72.

18. Nikica G, Ljerka HP, Jelena P, Metez-Soldo K, Mladen B. Cystoid macular edema in anterior chamber lens implantation following posterior capsule rupture. Doc Ophthalmol. 1992;81:309-15.

19. Ah-Fat FG, Sharma MK, Majid MA, Yang YC. Vitreous loss during conversion from conventional extracapsular cataract extraction to phacoemulsification. J Cataract Refract Surg. 1998;24:801-5.

20. Chinchurreta Capote AM, Lorenzo Soto M, Rivas Ruiz F, et al. Comparative study of the efficacy and safety of bromfenac, nepafenac and diclofenac sodium for the prevention of cystoid macular edema after phacoemulsification. Int J Ophthalmol. 2018;11:1210-6.

21. El Gharbawy SA, Darwish EA, Abu Eleinen KG, Osman MH. Efficacy of addition of nepafenac $0.1 \%$ to steroid eye drops in prevention of post-phaco macular edema in high-risk eyes. Eur J Ophthalmol. 2019; 29(4):453-7.

22. Baartman BJ, Gans R, Goshe J. Prednisolone versus dexamethasone for prevention of pseudophakic cystoid macular edema. Can J Ophthalmol. 2018;53:131-4.

23. Ylinen $P$, Holmstrom E, Laine I, Lindholm JM, Tuuminen R. Anti-inflammatory medication following cataract surgery: a randomized trial between preservative-free dexamethasone, diclofenac and their combination. Acta Ophthalmol. 2018;96:486-93.

24. Guo S, Patel S, Baumrind B, et al. Management of pseudophakic cystoid macular edema. Surv Ophthalmol. 2015;60:123-37.

25. Wielders LHP, Schouten J, Winkens B, van den Biggelaar FJHM, Veldhuizen $\mathrm{CA}$, Findl $\mathrm{O}$, et al. European multicenter trial of the prevention of cystoid macular edema after cataract surgery in nondiabetics: ESCRS PREMED study report 1. J Cataract Refract Surg. 2018;44:429-39. 
26. Feigenbaum A, Kornbluth W. Intravitreal injection of penicillin in a case of incipient abscess of the vitreous following extracapsular cataract extraction; perfect cure. Ophthalmologica. 1945;110:300-5.

27. Conway MD, Canakis C, Livir-Rallatos C, Peyman GA. Intravitreal triamcinolone acetonide for refractory chronic pseudophakic cystoid macula edema. J Cataract Refract Surg. 2003;29:27-33.

28. Benhamou N, Massin P, Haouchine B, Audren F, Tadayoni R, Gaudric A Intravitreal triamcinolone for refractory pseudophakic macular edema. Am $\mathrm{J}$ Ophthalmol 2003;135:246-9.

29. Boscia F, Furino C, Dammacco R, Ferreri P, Sborgia L, Sborgia C. Intravitreal triamcinolone acetonide in refractory pseudophakic cystoid macular edema: functional and anatomic results. Eur J Ophthalmol. 2005;15:89-95

30. Koutsandrea C, Moschos MM, Brouzas D, Loukianou E, Apostolopoulos M Moschos M. Intraocular triamcinolone acetonide for pseudophakic cystoid macular edema: optical coherence tomography and multifocal electroretinography study. Retina. 2007;27:159-64.

31. Lyall DA, Tey A, Foot B, Roxburgh ST, Virdi M, Robertson C, et al Post-intravitreal anti-VEGF endophthalmitis in the United Kingdom: incidence, features, risk factors, and outcomes. Eye (Lond). 2012;26:1517-26.

32. Poku E, Rathbone J, Wong R, Everson-Hock E, Essat M, Pandor A, et al. The safety of intravitreal bevacizumab monotherapy in adult ophthalmic conditions: systematic review. BMJ Open. 2014;4:e005244.

33. Fung AE, Rosenfeld PJ, Reichel E. The International Intravitreal Bevacizumab Safety Survey: using the internet to assess drug safety worldwide. Br J Ophthalmol. 2006:90:1344-9.

34. Arikan G, Osman Saatci A, Hakan Oner F. Immediate intraocular pressure rise after intravitreal injection of ranibizumab and two doses of triamcinolone acetonide. Int J Ophthalmol. 2011:4:402-5.

35. Chan CK, Fan DS, Chan WM, Lai WW, Lee VY, Lam DS. Ocular-hypertensive response and corneal endothelial changes after intravitreal triamcinolone injections in Chinese subjects: a 6-month follow-up study. Eye (Lond). 2005:19:625-30.

36. Veritti D, Di Giulio A, Sarao V, Lanzetta P. Drug safety evaluation of intravitreal triamcinolone acetonide. Expert Opin Drug Saf 2012;11:331-40.

37. Altamirano-Vallejo JC, Navarro-Partida J, Gonzalez-De la Rosa A Hsiao JH, Olguín-Gutierrez JS, Gonzalez-Villegas AC, et al. Characterization and Pharmacokinetics of Triamcinolone Acetonide-Loaded Liposomes Topical Formulations for Vitreoretinal Drug Delivery. J Ocul Pharmacol Ther. 2018:34:416-25.

38. Gonzalez-De la Rosa A, Navarro-Partida J, Altamirano-Vallejo JC, Hernandez-Gamez AG, Garcia-Bañuelos JJ, Armendariz-Borunda J, et al Novel Triamcinolone Acetonide-Loaded Liposomes Topical Formulation for the Treatment of Cystoid Macular Edema After Cataract Surgery: A Pilot Study. J Ocul Pharmacol Ther. 2019;35(2):106-15.

39. Gonzalez-De la Rosa A, Navarro-Partida J, Altamirano-Vallejo JC, Jauregui-Garcia GD, Acosta-Gonzalez R, Ibanez-Hernandez MA, et al. Nove Triamcinolone Acetonide-Loaded Liposomal Topical Formulation Improves Contrast Sensitivity Outcome After Femtosecond Laser-Assisted Cataract Surgery. J Ocul Pharmacol Ther. 2019;35(9):512-21.

40. Levin DS, Lim JI. Update on pseudophakic cystoid macular edema treatment options. Ophthalmol Clin North Am. 2002;15:467-72.

41. Heier JS, Topping TM, Baumann W, Dirks MS, Chern S. Ketorolac versus prednisolone versus combination therapy in the treatment of acute pseudophakic cystoid macular edema. Ophthalmology. 2000;107: 2034-2038; discussion 2039
42. Meyer LM, Schonfeld CL. Cystoid Macular Edema after Complicated Cataract Surgery Resolved by an Intravitreal Dexamethasone 0.7-mg Implant. Case Rep Ophthalmol. 2011;2:319-22.

43. Rho DS. Treatment of acute pseudophakic cystoid macular edema: Diclofenac versus ketorolac. J Cataract Refract Surg. 2003;29:2378-84.

44. Warren KA, Fox JE. Topical nepafenac as an alternate treatment for cystoid macular edema in steroid responsive patients. Retina. 2008:28:1427-34

45. Warren KA, Bahrani $H$, Fox JE. NSAIDs in combination therapy for the treatment of chronic pseudophakic cystoid macular edema. Retina. 2010;30:260-6.

46. Zhang X, Wang N, Schachat AP, et al. Glucocorticoids: structure, signaling and molecular mechanisms in the treatment of diabetic retinopathy and diabetic macular edema. Curr Mol Med. 2014;14:376-84.

47. Ebrahem Q, Minamoto A, Hoppe G, Anand-Apte B, Sears JE. Triamcinolone acetonide inhibits IL-6- and VEGF-induced angiogenesis downstream of the IL-6 and VEGF receptors. Invest Ophthalmol Vis Sci. 2006;47:4935-41.

48. Kwon SI, Kim YW, Bang YW, Lee JY, Park IW. Comparison of natural course, intravitreal triamcinolone, and intravitreal bevacizumab for treatment of macular edema secondary to branch retinal vein occlusion. $J$ Ocul Pharmacol Ther. 2013;29:5-9.

49. Habot-Wilner Z, Sallam A, Pacheco PA, Do HH, McCluskey P, Lightman $S$, et al. Intravitreal triamcinolone acetonide as adjunctive treatment with systemic therapy for uveitic macular edema. Eur J Ophthalmol. 2011;21 Suppl 6:S56-61

50. Yalcinbayir O, Gelisken O, Kaderli B, Avci R. Intravitreal versus sub-tenon posterior triamcinolone injection in bilateral diffuse diabetic macular edema. Ophthalmologica. 2011;225:222-7.

51. Shrivastava A, Singh K. The effect of cataract extraction on intraocular pressure. Curr Opin Ophthalmol. 2010;21:118-22.

52. Slabaugh MA, Chen PP. The effect of cataract extraction on intraocular pressure. Curr Opin Ophthalmol. 2014;25:122-6.

53. Klibanov AL, Maruyama K, Torchilin VP, Huang L. Amphipathic polyethyleneglycols effectively prolong the circulation time of liposomes. FEBS Lett. 1990:268:235-7.

54. Lopez-Berestein G, Mehta R, Hopfer R, Mehta K, Hersh EM, Juliano R. Effects of sterols on the therapeutic efficacy of liposomal amphotericin $B$ in murine candidiasis. Cancer Drug Deliv. 1983;1:37-42.

55. Oku N, Nojima S, Inoue K. Selective release of non-electrolytes from liposomes upon perturbation of bilayers by temperature change or polyene antibiotics. Biochim Biophys Acta 1980:595:277-90.

56. Allen TM, Cullis PR. Drug delivery systems: entering the mainstream. Science 2004;303:1818-22.

57. van Rooijen N, van Nieuwmegen R. Liposomes in immunology: multilamellar phosphatidylcholine liposomes as a simple, biodegradable and harmless adjuvant without any immunogenic activity of its own. Immunol Commun 1980;9:243-56.

58. Di Tommaso C, Bourges ل JL, Valamanesh F, Trubitsyn G, Torriglia A Jeanny JC, et al. Novel micelle carriers for cyclosporin A topical ocular delivery: in vivo cornea penetration, ocular distribution and efficacy studies. Eur J Pharm Biopharm. 2012;81:257-64.

59. Hathout RM, Mansour S, Mortada ND, Guinedi AS. Liposomes as an ocular delivery system for acetazolamide: in vitro and in vivo studies. AAPS PharmSciTech. 2007;8:1. 